\title{
Thromboembolism in children with multisystem inflammatory syndrome: a literature review
}

\author{
Neethu M. Menon ${ }^{1 凶}$ and Lakshmi V. Srivaths ${ }^{1}$ \\ (c) The Author (s), under exclusive license to the International Pediatric Research Foundation, Inc. 2021
}

\begin{abstract}
Multisystem inflammatory syndrome in children (MIS-C) is a hyperinflammatory response observed in children several weeks to months after acute infection with severe acute respiratory syndrome coronavirus-2 (SARS-CoV-2). On review of all published cases of thromboembolism (TE) as a complication of MIS-C, 33 cases of TE were found with incidence ranging from 1.4 to $6.5 \%$. TE occurred mostly in children aged 12 years and above. One-third of the cases were cerebral infarcts and the remaining cases included intracardiac and radial arterial thromboses, upper and lower extremity deep vein thrombosis, pulmonary embolism, and splenic infarcts. Five were asymptomatic cases and 3/33 (9\%) patients (all three with cerebral infarcts) died. To conclude, TE appears to be a significant complication of MIS-C caused by SARS-CoV- 2 infection, associated with morbidity and/or mortality. Patients $\geq 12$ years are affected more often, and TE occurs despite thromboprophylaxis in some patients. Thromboprophylaxis should be considered in all cases after reviewing the concomitant bleeding risk. Prospective studies are needed to confirm the role of standard-dose thromboprophylaxis and to explore whether higher-dose thromboprophylaxis is required in certain high-risk patients with MIS-C.
\end{abstract}

Pediatric Research (2022) 92:946-950; https://doi.org/10.1038/s41390-021-01873-0

IMPACT:

- Compiles all cases of thromboembolism associated with COVID-19-related MIS-C, a report that has not been published to date.

\section{INTRODUCTION}

Within a few months of the worldwide spread of severe acute respiratory syndrome coronavirus-2 (SARS-CoV-2) infection, a hyperinflammatory response to the infection was observed in children, occurring several weeks to months after the acute infection. The terminology multisystem inflammatory syndrome of childhood (MISC) was coined to describe this disease process. ${ }^{1}$ First published in April 2020 with the occurrence of fever, cardiovascular instability, and a diffuse inflammatory process in eight children with positive antibody tests for SARS-COV-2, MIS-C has now been reported all across the world. ${ }^{2}$ It is broadly defined as an inflammatory condition affecting the cardiovascular, pulmonary, hematologic, renal, gastrointestinal, and/or neurologic systems. It often presents as fever along with variable symptoms such as abdominal pain, emesis, diarrhea, skin rash, and generalized fatigue. The World Health Organization (WHO) and Centers for Disease Control and Prevention (CDC) case definitions of MIS-C are outlined in Table 1.,4

The increased risk of thrombosis with coronavirus disease 2019 (COVID-19) in adults as well as in children has been described in several studies. ${ }^{5,6}$ The clinical manifestations of MIS-C have also been systematically reviewed by multiple researchers across the world, but the risk of thromboembolism (TE) related to MIS-C specifically has not been reviewed to date. The purpose of this review is to consolidate reports of TE events in children with MIS-C related to SARS-CoV-2 infection with the description of different types of TE events reported, associated laboratory features, and management approaches.

\section{METHODS}

The investigators searched PUBMED and EMBASE on April 19, 2021 using the search term "multisystem inflammatory syndrome AND COVID-19" for years 2020 and 2021. The article type "Books and Documents" was excluded in PUBMED filters. For EMBASE, the search was filtered to include "Article" and "Article in Press" under "Publication types" and the category "pediatric multisystem inflammatory syndrome" under "Disease." Reports and reviews of MIS-C without any information regarding TE cases, and guidance/recommendation statements or protocols without TE case information were excluded. Publications in languages other than English were also excluded. The full texts of potentially eligible publications were then reviewed in detail. Since some of the earlier studies may have been missed if they were not reported using the specific terminology, relevant references found in each of these studies that met the inclusion and exclusion criteria were also reviewed.

\section{RESULTS}

The initial search showed 162 articles on EMBASE and 167 articles on PUBMED, 12 of which were common to both, leaving 317 articles related to MIS-C (Fig. 1). Two hundred and eighty-seven articles were excluded based on the absence of any information regarding cases of $\mathrm{TE}, 15$ articles were guidance statements or protocols, and 6 articles were in languages other than English. The remaining nine studies met the inclusion and exclusion criteria.

\footnotetext{
${ }^{1}$ Division of Hematology, Department of Pediatrics, University of Texas Health Science Center at Houston, 7000 Fannin Street, Houston, TX 77030, USA.

email: Neethu.m.menon@uth.tmc.edu
}

Received: 12 May 2021 Revised: 13 September 2021 Accepted: 13 November 2021

Published online: 14 January 2022 
Table 1. Case definition of MIS-C.

\section{WHO, May $2020^{3}$}

$0-19$ years of age with fever $\geq 3$ days AND 2 of the following

-rash or bilateral nonpurulent conjunctivitis or mucocutaneous inflammation -hypotension or shock

-features of myocardial dysfunction, pericarditis, valvulitis, or coronary abnormalities

-coagulopathy

-acute gastrointestinal symptoms

AND elevated markers of inflammation

AND no other obvious microbial cause

AND evidence of COVID-19 or contact with it

\section{CDC, June $2020^{4}$}

0-21 years of age with fever, laboratory evidence of inflammation, and evidence of clinically severe illness requiring hospitalization, with multisystem $(>2)$ organ involvement

AND no alternative plausible diagnosis

AND positive for current or recent SARS-CoV-2 infection or exposure to a suspected or confirmed COVID-19 case within 4 weeks

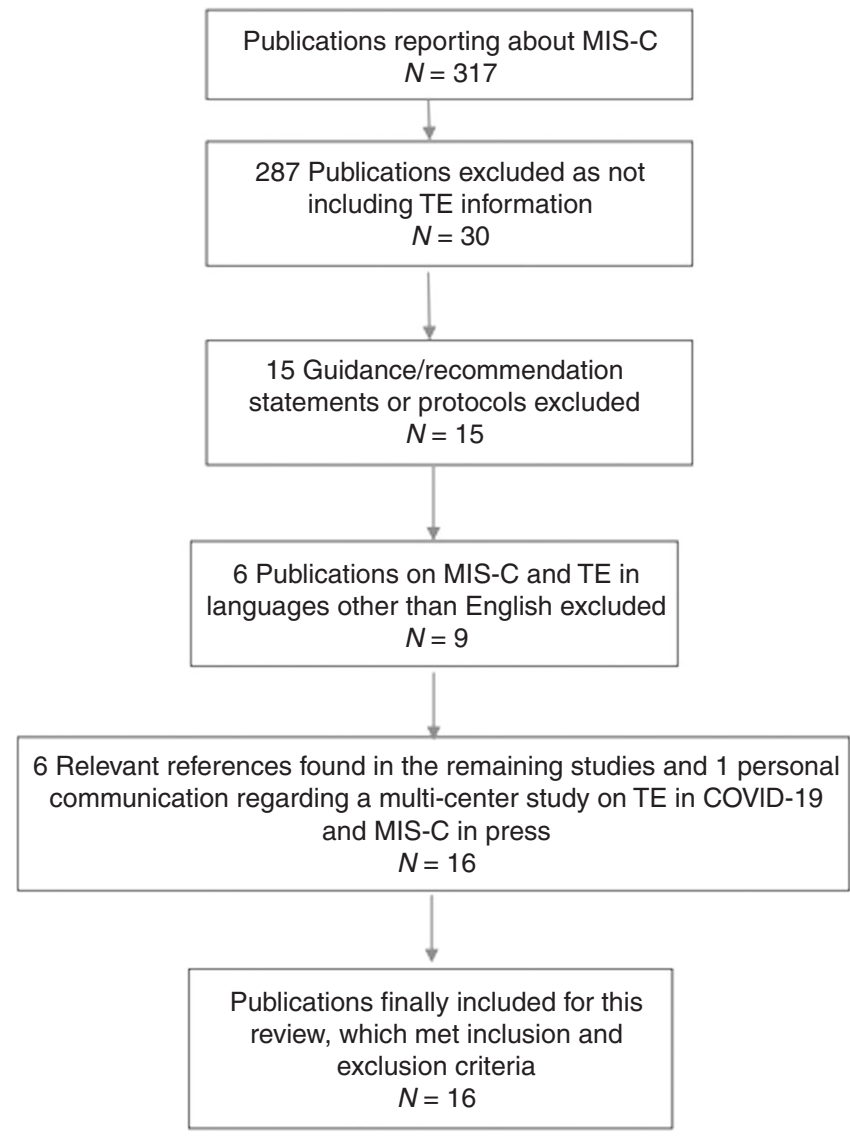

Fig. 1 Search flowchart showing the publications searched and reviewed based on inclusion and exclusion criteria. MIS-C multisystem inflammatory syndrome in children. TE thromboembolism.

Including six relevant references found in these studies and personal communication regarding a multicenter study on TE in COVID-19 and MIS-C in the press, a total of 16 studies found to have data on TE in MIS-C related to COVID-19 were finally included for the purpose of this review. Although there were several systematic reviews on the initial search, data pertinent to TE was only found in three. ${ }^{7-9}$ Other cases of TE were found in single and multicenter observational studies, case series, and case reports (Table 2).

The earliest case of TE was reported in the first published MIS-C case series, in a 14-year-old boy who developed acute ischemic stroke with infarction of right anterior and middle cerebral arteries while on extracorporeal membrane oxygenation (ECMO) for acute multiorgan dysfunction, despite being on anticoagulation with unfractionated heparin (UFH); this patient did not survive. ${ }^{2}$ The remaining seven children in this series did not have TE. The same center reported two patients with TE in MIS-C cases on ECMO, including the case already described above by Riphagen et al. ${ }^{2}$ and another child developing a right atrial thrombus despite being on high-dose aspirin and UFH with a level higher than their center's typical interquartile anti-factor $\mathrm{Xa}$ range; the thrombus resolved in $48 \mathrm{~h}$ without any long-term sequelae. ${ }^{10}$

The largest observational cohort reporting TE is a multicenter study from New York, United States (USA) with 4/186 (3.3\%) patients with MIS-C developing either deep vein thrombosis (DVT) or pulmonary embolism (PE). This included $1 / 75$ children aged $5-12$ years (1\%) and 3/45 aged $12-20$ years $(7 \%)$, with the site of TE not reported. ${ }^{11}$ Although many patients received anticoagulant therapy including UFH, low molecular weight heparin (LMWH), bivalirudin, warfarin, and argatroban, thromboprophylaxis, management, and outcome of the four patients with TE were not separately reported. An intensive care unit (ICU) cohort from the United Kingdom (UK) reported three TE cases in 78 children with MIS-C (3.8\%), all of whom received therapeutic anticoagulation, with site and outcome not described. ${ }^{12}$ In this study, $41 \%$ of patients received prophylactic anticoagulation, but the thromboprophylaxis received by the patients developing TE is not reported.

The eight venous TE (VTE) cases out of 229 patients (3.4\%) described by Aronoff et al. in their systematic review of 16 studies have all been described above (the case of right atrial thrombus was not included in this review). ${ }^{7}$ Hoste et al reviewed 68 studies and reported five additional cases hence reporting a total of 13 cases of TE in 953 MIS-C patients (1.4\%). ${ }^{8}$ Two of these were splenic infarcts detected on imaging without corresponding thrombi in the abdominal vasculature and their anticoagulation management is not reported. ${ }^{13}$ The three remaining cases were acute cerebral infarcts in critically ill patients on ECMO and two of these patients died. ${ }^{13,14}$ Anticoagulation details are not reported, but at least one of them received UFH while on ECMO prior to the occurrence of the infarct. Heparin (type not specified) was reported as the most commonly used anticoagulant agent (46\%) by Hoste et al. with prophylactic versus therapeutic dosing not specified. Tang et al. reviewed 24 studies and reported the case already reported above by Riphagen et al. ${ }^{9}$

Another patient placed on ECMO (within $24 \mathrm{~h}$ of presentation) is described in a New York study of 33 MIS-C cases, who developed an ischemic brain infarct on day 6 of ECMO, and died due to hemorrhagic stroke. ${ }^{15}$ In this study, all patients received either prophylactic enoxaparin or therapeutic anticoagulation with enoxaparin or UFH. Two studies from India described central nervous system infarcts in one MIS-C patient each, with clinical improvement in both cases; both of these patients were not on ECMO but had a prolonged stay in the pediatric ICU (>7 days). ${ }^{16,17}$ One patient was treated with a therapeutic dose of enoxaparin. One of these studies by Shobavat et al. also reported two cases of 


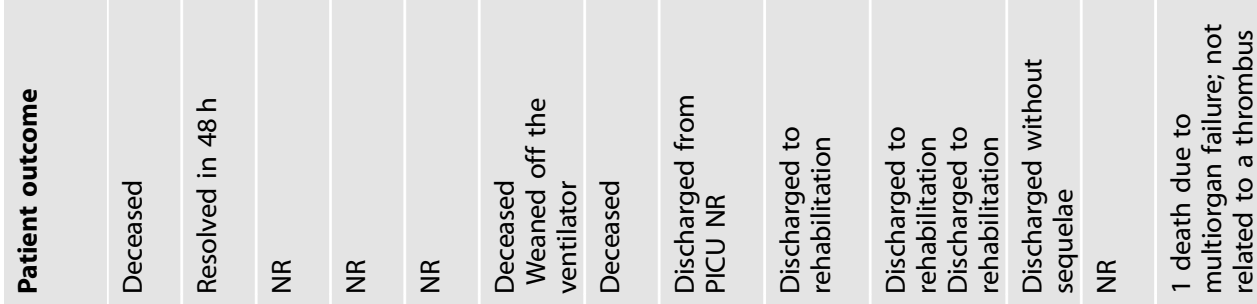

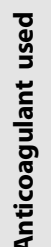

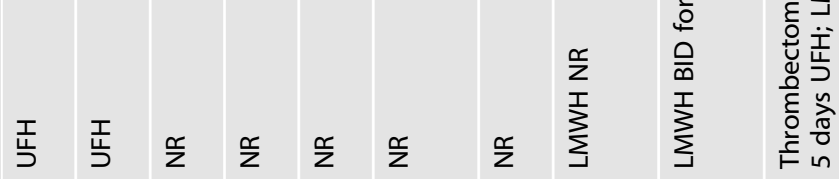

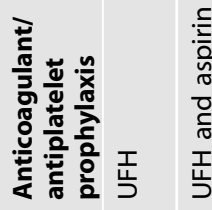

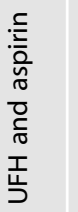

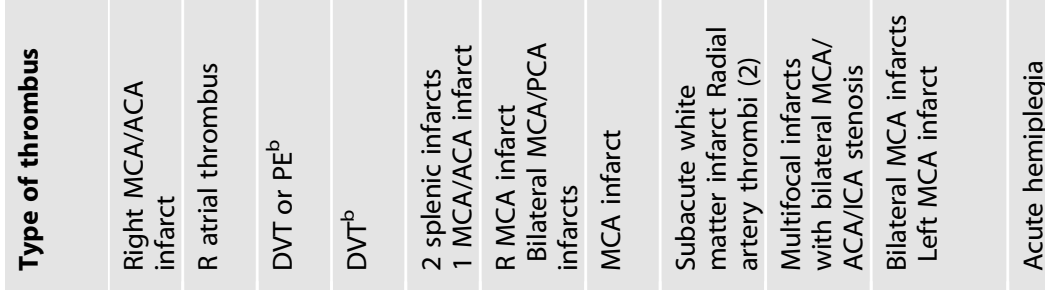

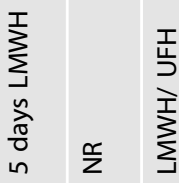

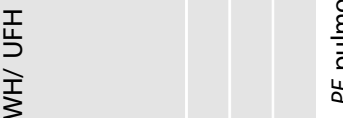

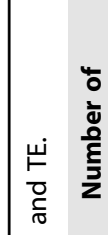

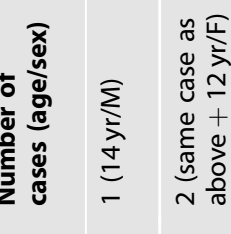

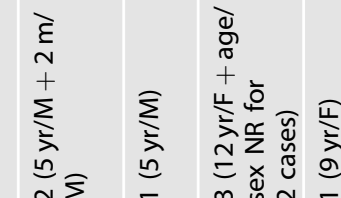

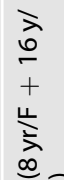

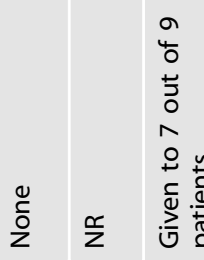

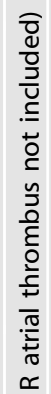

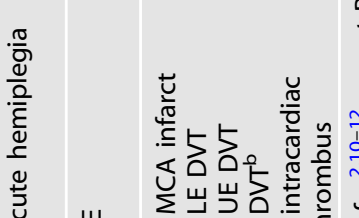

ํํำ

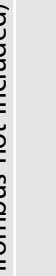

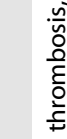

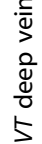

$\stackrel{\omega}{\Sigma}$

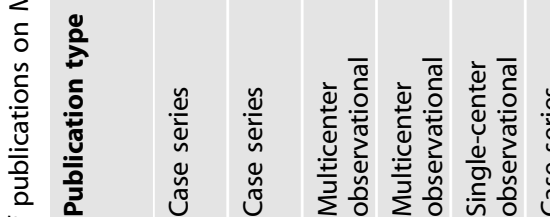

N 2

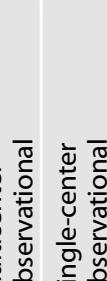

$$
N-
$$

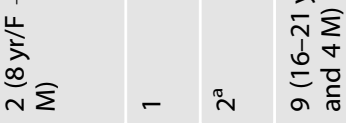
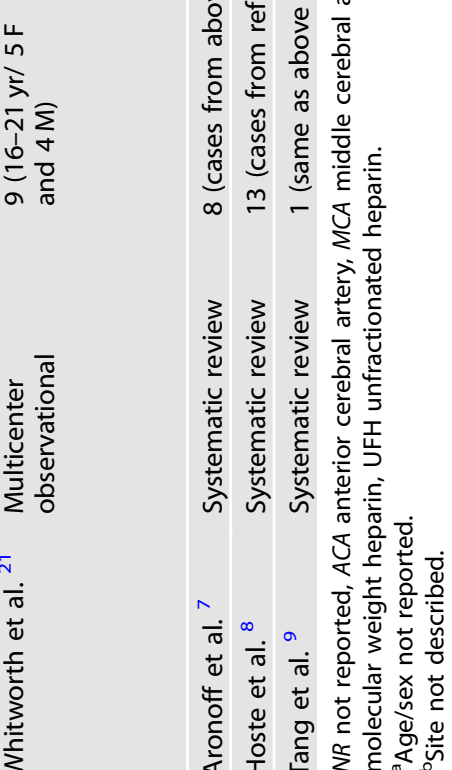
radial artery thrombosis related to arterial line placement; both resolved with therapeutic anticoagulation. ${ }^{16}$ More recently, two cases of acute onset hemiparesis were described from the Southwest US, 3-4 weeks after acute symptoms of COVID-19, both confirmed to be severe cerebral infarcts. ${ }^{18}$ One was an 8year-old girl with positive COVID-19 antibodies, anticoagulated (agent not specified) for 5 days followed by a transition to aspirin, and the other was a 14-year-old boy with a positive COVID-19 polymerase chain reaction test from the time of initial symptoms, treated with UFH followed by enoxaparin (duration not reported). ${ }^{18}$ Acute hemiparesis, along with fever and a diffuse rash, is also described in a 6-year-old boy in India with positive COVID-19 antibodies, not confirmed by imaging to be infarcts. ${ }^{19}$ $\mathrm{He}$ received enoxaparin, intravenous immunoglobulin, and steroids with complete resolution of symptoms in 3 days without any residual neurological deficits.

A retrospective records review of imaging studies in 16 MIS-C patients at a New York center found two positive cases out of eight children imaged for suspected PE; management details were not included. ${ }^{20}$

Most recently, a US multicenter study retrospectively reviewed the incidence of thrombosis in pediatric COVID-19 cases and found 9 out of 138 MIS-C cases $(6.5 \%)$ with thrombotic events. ${ }^{21}$ Central venous catheter placement was an additional risk factor present in every case. The patients who developed thrombosis were on prophylactic anticoagulation with either enoxaparin or UFH, with the exception of the child with a cerebral infarct on admission and a critically ill child admitted in shock. There were seven cases of DVT (two asymptomatic), one cerebral infarct in a critically ill patient admitted with stroke symptoms, and one intracardiac thrombus occurring postECMO. One patient with asymptomatic DVT died due to multiorgan failure, unrelated to thrombosis.

\section{DISCUSSION}

The overall incidence of TE in children is low compared to adults. ${ }^{22}$ As the COVID-19 pandemic surged, children were observed to have milder courses; in turn, when the prothrombotic risk of SARSCoV-2 was recognized, this also appeared to be less concerning in the pediatric population. ${ }^{23}$ However, when MIS-C started to occur in children, pediatric TE cases began to be reported from centers all across the world.

This review summarizes the reports of 33 cases of pediatric TE found in patients with MIS-C. Of these, there were ten males and nine females, with sex not reported in the remaining cases. Onethird of these were cerebral infarcts and the remaining included intracardiac (2) and radial arterial (2) clots, upper extremity (4) and lower extremity (2) DVT, PE (2), and splenic infarcts (2), with the site not described in remaining cases. Treatment details were not available in most cases (24), but included heparin (UFH, $N=5$; LMWH, $N=4$ ), thrombectomy for a patient with MCA infarct, and aspirin in four cases of cerebral infarcts. Three deaths reported were in patients on ECMO with cerebral infarcts and the fourth death was unrelated to thrombosis. Thromboprophylaxis details were available for $13 / 33$ cases ( 11 received prophylaxis) with the more recent studies reporting the use of UFH or LMWH in most cases likely due to the increased awareness over time of the risk of TE in MIS-C.

A lower incidence of around $1.4-4 \%$ of MIS-C-related thrombosis reported in most initial studies versus a higher incidence of $6.5 \%$ overall and $19 \%$ in children aged 12 years and above in the most recent multicenter study ${ }^{21}$ is likely due to a high index of clinical suspicion as the entity was better defined, with more frequent and thorough evaluation for TE over time. With no standardized method of prophylactic anticoagulation, it is difficult to compare these studies or even patients within each study. However, it is noteworthy that seven out of nine cases reported by Whitworth et al. developed TE while on thromboprophylaxis with some form of heparin. ${ }^{21}$ Aronoff et al. also reported more than half of the patients in their systematic review to be on prophylactic anticoagulation. $^{7}$

Nineteen out of the 28 cases (68\%) where age was reported occurred in children aged 12 years and above and Whitworth et al. reported a high MIS-C-related TE incidence of $19 \%$ in this age group. Hence, thromboprophylaxis in this age group of MIS-C cases appears most important.

Most cases occurred in critically ill patients with multiple comorbidities with central venous catheter identified as an additional risk factor by one study. ${ }^{21}$ It is noteworthy that $8 / 33$ patients (24.2\%) were on ECMO with three fatal outcomes. Although there is a well-known risk of stroke with ECMO, increased VTE has been reported with COVID-19 and ECMO when compared to ECMO for other causes. ${ }^{24}$ Ischemic stroke has been reported with COVID-19 with or without ECMO. ${ }^{25}$ In the patients developing stroke while on ECMO, both ECMO and COVID-19 likely contributed to the stroke.

The reports of cerebral infarcts are intriguing because some of these are associated with intracranial hemorrhage, making it hard to ascertain whether the inciting insult was ischemic or hemorrhagic. Regardless, its occurrence has a poor prognosis with 3 out of the 11 reported patients failing to survive.

The limitation of our review is the incomplete data on prophylaxis, management, and outcome of TE cases and this is primarily due to its retrospective nature. Nevertheless, it provides valuable information about the types of TE seen in MIS-C, affected age groups, and the occurrence of TE despite thromboprophylaxis.

To conclude, based on our review, TE appears to be a significant complication of MIS-C associated with SARS-CoV-2 infection in children, with a higher incidence in children 12 years and older, and appears to occur despite thromboprophylaxis, indicating a high risk of thrombosis in this patient population. Patients with MIS-C and TE appear to have significant morbidity and/or mortality. Based on these results, it appears reasonable to consider thromboprophylaxis in children with MIS-C, after careful review of the concomitant bleeding risk. Prospective studies are needed to look at the impact of standard-dose prophylactic anticoagulation in MIS-C and the need for higher-dose prophylactic anticoagulation in select high-risk patients, in preventing TE in MIS-C.

\section{REFERENCES}

1. Henderson, L. A. et al. American College of Rheumatology Clinical Guidance for Multisystem Inflammatory Syndrome in Children associated with SARS-CoV-2 and hyperinflammation in pediatric COVID-19: version 1. Arthritis Rheumatol. 72, 1791-1805 (2020).

2. Riphagen, S., Gomez, X., Gonzalez-Martinez, C., Wilkinson, N. \& Theocharis, P. Hyperinflammatory shock in children during COVID-19 pandemic. Lancet 395 1607-1608 (2020).

3. World Health Organization. Multisystem Inflammatory Syndrome in Children and Adolescents with COVID-19: Scientific Brief 15 May 2020 (World Health Organization, 2020).

4. Centers for Disease Control and Prevention. Reporting Multisystem Inflammatory Syndrome in Children (MIS-C) (Centers for Disease Control and Prevention, 2020).

5. Nopp, S., Moik, F., Jilma, B., Pabinger, I. \& Ay, C. Risk of venous thromboembolism in patients with COVID-19: a systematic review and meta-analysis. Res. Pract. Thromb. Haemost. 4, 1178-91 (2020).

6. Del Borrello, G. et al. SARS-COV-2-associated coagulopathy and thromboembolism prophylaxis in children: a single-center observational study. J. Thromb. Haemost. 19, 522-30. (2021).

7. Aronoff, S. C., Hall, A., Del \& Vecchio, M. T. The natural history of severe acute respiratory syndrome coronavirus 2-related multisystem inflammatory syndrome in children: a systematic review. J. Pediatr. Infect. Dis. Soc. 9, 746-51. (2020).

8. Hoste, L., Van Paemel, R. \& Haerynck, F. Multisystem inflammatory syndrome in children related to COVID-19: a systematic review. Eur. J. Pediatr. 180, 2019-34 (2021).

9. Tang, Y. et al. Multisystem inflammatory syndrome in children during the coronavirus disease 2019 (COVID-19) pandemic: a systematic review of published case studies. Transl. Pediatr. 10, 121-35. (2021). 
10. Minen, F., Hands, C., Mustafa, M. R., Pienaar, A. \& Lillie, J. Thrombophilia in pediatric patients with multisystem inflammatory syndrome in children secondary to coronavirus disease 2019 supported on extracorporeal membrane oxygenation. ASAIO J. 67, 7-11 (2021).

11. Feldstein, L. R. et al. Multisystem inflammatory syndrome in U.S. children and adolescents. N. Engl. J. Med. 383, 334-46. (2020).

12. Davies, P. et al. Intensive care admissions of children with paediatric inflammatory multisystem syndrome temporally associated with SARS-CoV-2 (PIMS-TS) in the UK: a multicentre observational study. Lancet Child Adolesc. Health 4, 669-77. (2020).

13. Hameed, S. et al. Spectrum of imaging findings at chest radiography, US, CT, and $\mathrm{MRI}$ in multisystem inflammatory syndrome in children associated with COVID19. Radiology 298, E1-E10 (2021).

14. Schupper, A. J., Yaeger, K. A. \& Morgenstern, P. F. Neurological manifestations of pediatric multi-system inflammatory syndrome potentially associated with COVID-19. Childs Nerv. Syst. 36, 1579-80. (2020).

15. Kaushik, S. et al. Multisystem inflammatory syndrome in children associated with severe acute respiratory syndrome coronavirus 2 infection (MIS-C): A MultiInstitutional Study from New York City. J. Pediatr. 224, 24-29 (2020).

16. Shobhavat, L. et al. Multisystem inflammatory syndrome in children: clinical features and management-intensive care experience from a pediatric public hospital in western India. Indian J. Crit. Care Med. 24, 1089-94. (2020).

17. Tiwari, L., Shekhar, S., Bansal, A. \& Kumar, S. COVID-19 associated arterial ischaemic stroke and multisystem inflammatory syndrome in children: a case report. Lancet Child Adolesc. Health 5, 88-90 (2021).

18. Appavu, B. et al. Arteritis and large vessel occlusive strokes in children after COVID-19 infection. Pediatrics 147, e2020023440 (2021)

19. Thomas, A. Novel presentation of pediatric inflammatory multisystem syndrome temporally associated with COVID-19. J. Pediatr. Neurol. https://doi.org/10.1055/s0041-1725982 (2021).

20. Blumfield, E., Levin, T. L., Kurian, J., Lee, E. Y. \& Liszewski, M. C. Imaging findings in multisystem inflammatory syndrome in children (MIS-C) associated with coronavirus disease (COVID-19). Am. J. Roentgenol. 216, 507-17. (2021).

21. Whitworth, H. B. et al. Rate of thrombosis in children and adolescents hospitalized with COVID-19 or MIS-C. Blood 138, 190-8 (2021).

22. Chalmers, E. A. Epidemiology of venous thromboembolism in neonates and children. Thromb. Res. 118, 3-12 (2006).

23. Goldenberg, N. A. et al. Consensus-based clinical recommendations and research priorities for anticoagulant thromboprophylaxis in children hospitalized for COVID-19-related illness. J. Thromb. Haemost. 18, 3099-105. (2020).
24. Doyle, A. J. et al. A comparison of thrombosis and hemorrhage rates in patients with severe respiratory failure due to coronavirus disease 2019 and influenza requiring extracorporeal membrane oxygenation. Crit. Care Med. 49, e663-e72. (2021).

25. Kleineberg, N. N. et al. Neurological symptoms and complications in predominantly hospitalized COVID-19 patients: results of the European multinational Lean European Open Survey on SARS-Infected Patients (LEOSS). Eur. J. Neurol. 28 3925-37 (2021).

\section{AUTHOR CONTRIBUTIONS}

L.V.S. contributed to the conception of the study, data acquisition, analysis and interpretation, revision, and approval of the final version of the manuscript. N.M.M. contributed to the design of the study, data acquisition, analysis and interpretation, drafting of the manuscript, and approval of the final version of the manuscript.

\section{FUNDING}

No financial assistance was received in support of this study. There are no perceived conflicts of interest for any of the authors and the authors do not have financial ties to any products in the study.

\section{COMPETING INTERESTS}

The authors declare no competing interests.

\section{ADDITIONAL INFORMATION}

Correspondence and requests for materials should be addressed to Neethu $M$. Menon.

Reprints and permission information is available at http://www.nature.com/ reprints

Publisher's note Springer Nature remains neutral with regard to jurisdictional claims in published maps and institutional affiliations. 